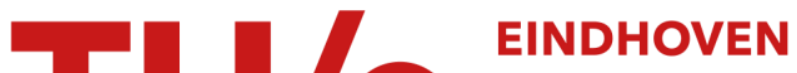 \\ UNIVERSITY OF \\ TECHNOLOGY
}

\section{Termination by quasi-periodic interpretations}

Citation for published version (APA):

Zantema, H., \& Waldmann, J. (2007). Termination by quasi-periodic interpretations. In F. Baader (Ed.), Proceedings of the 18th International Conference on Term Rewriting and Applications (RTA 2007) 26-28 June 2007, Paris, France (pp. 404-418). (Lecture Notes in Computer Science; Vol. 4533). Springer.

https://doi.org/10.1007/978-3-540-73449-9_30

DOI:

10.1007/978-3-540-73449-9_30

Document status and date:

Published: 01/01/2007

\section{Document Version:}

Publisher's PDF, also known as Version of Record (includes final page, issue and volume numbers)

\section{Please check the document version of this publication:}

- A submitted manuscript is the version of the article upon submission and before peer-review. There can be important differences between the submitted version and the official published version of record. People interested in the research are advised to contact the author for the final version of the publication, or visit the $\mathrm{DOI}$ to the publisher's website.

- The final author version and the galley proof are versions of the publication after peer review.

- The final published version features the final layout of the paper including the volume, issue and page numbers.

Link to publication

\section{General rights}

Copyright and moral rights for the publications made accessible in the public portal are retained by the authors and/or other copyright owners and it is a condition of accessing publications that users recognise and abide by the legal requirements associated with these rights.

- Users may download and print one copy of any publication from the public portal for the purpose of private study or research.

- You may not further distribute the material or use it for any profit-making activity or commercial gain

- You may freely distribute the URL identifying the publication in the public portal.

If the publication is distributed under the terms of Article 25fa of the Dutch Copyright Act, indicated by the "Taverne" license above, please follow below link for the End User Agreement:

www.tue.nl/taverne

Take down policy

If you believe that this document breaches copyright please contact us at:

openaccess@tue.nl

providing details and we will investigate your claim. 


\title{
Termination by Quasi-periodic Interpretations
}

\author{
Hans Zantema ${ }^{1}$ and Johannes Waldmann ${ }^{2}$ \\ ${ }^{1}$ Department of Computer Science, Technische Universiteit Eindhoven \\ P.O. Box 513, 5600 MB Eindhoven, The Netherlands \\ h.zantema@tue.nl \\ ${ }^{2}$ Hochschule für Technik, Wirtschaft und Kultur (FH) Leipzig \\ Fb IMN, PF 3011 66, D-04251 Leipzig, Germany \\ waldmann@imn.htwk-leipzig.de
}

\begin{abstract}
We present a new method for automatically proving termination of term rewriting and string rewriting. It is based on the wellknown idea of interpretation of terms in natural numbers where every rewrite step causes a decrease. In the dependency pair setting only weak monotonicity is required for these interpretations. For these we use quasiperiodic functions. It turns out that then the decreasingness for rules only needs to be checked for finitely many values, which is easy to implement.

Using this technique we automatically prove termination of over ten string rewriting systems in TPDB for which termination was open until now.
\end{abstract}

\section{Introduction}

At the Workshop on Termination, Seattle, August 2006, termination of the string rewriting system (SRS) consisting of the two rules

$$
a a a \rightarrow b a b, \quad b b b \rightarrow a a a,
$$

was presented as an open problem. Shortly after that Aleksey Nogin and Carl Witty came up with an ad hoc proof, based on observations on the length modulo 3 of maximal numbers of consecutive $a$ 's in the string. Inspired by this proof based on this modulo 3 behavior, we found alternative termination proofs for the same SRS using dependency pairs and weakly monotone interpretations of the shape

$$
f(x)=3 *(x \operatorname{div} 3)=x-(x \bmod 3) .
$$

In this way one challenge for the current paper was born: generalize this kind of functions in such a way that they are suitable for implementation, and that using these functions increases the power of termination provers.

During the last years proving termination of small SRSs received a lot of attention, see e.g. [14/5,13,10,6]8]. Among the reasons for this we mention:

- It is an open problem whether termination of one rule string rewriting is decidable or not. Motivated by this open problem people are triggered to investigate termination behavior of single rules. 
- There are several extremely small SRSs like $\{a a b b \rightarrow b b b a a a\}$ and $\{a a \rightarrow$ $b c, b b \rightarrow a c, c c \rightarrow a b\}$ for which proving termination turned out to be surprisingly hard. For these two examples termination proofs are known and can be found by tools, but for several others the termination problem is still open.

Every year there is a Termination Competition 9, where several tools are applied on the newest version of the Termination Problem Data Base (TPDB) 2. In the string rewriting category of the 2006 version of TPDB there are 34 systems for which in the 2006 termination competition none of the tools could prove termination or non-termination, some of which are known to be nonterminating. By our implementation of the technique presented in this paper for 13 among these 34 systems termination proofs are generated fully automatically.

Our approach is an instance of the well-known theme of interpretations into a well-founded monotone algebra. It is well-known that in the setting of dependency pairs one only needs weak monotonicity in the algebra. In this paper we focus on the case where the monotone algebra simply consists of the natural numbers with the usual order. So the key point is to choose suitable weakly monotonic functions. In the usual approach these functions are always polynomials. Instead we choose functions that have a periodic difference with linear functions, more precisely, functions $f$ satisfying

$$
f(x+p)=f(x)+s * p
$$

for all $x \in \mathbf{N}$, for some period $p \in \mathbf{N}, p>1$ and some slope $s \in \mathbf{N}$. Such functions are called quasi-periodic. Note that the function $f$ mentioned above given by $f(x)=3 *(x \operatorname{div} 3)$ is an instance of a quasi-periodic function with period 3 and slope 1 . Moreover, all linear polynomial interpretations are quasiperiodic.

The following observations make quasi-periodic functions suitable for use in automatic search for termination proofs:

- A quasi-periodic function is fully determined by its slope, its period, and finitely many values.

- Quasi-periodic functions are closed under composition, by which the interpretation of a term is quasi-periodic if the interpretations of its operations symbols are.

- Checking whether $\forall x: f(x) \geq g(x)$ for quasi-periodic functions $f, g$ can be done by inspecting only finitely many values of $x$.

As a consequence, by fixing the period and bounding the slope the corresponding search space for function interpretations is finite. However, it may be huge. We have implemented the method by fixing the period and bounding the slope, transforming the search problem to a SAT problem, and using the stateof-the-art SAT solver minisat, version 2, 3. Surprisingly, all newly solved SRSs from TPDB could be solved by fixing the slope to be 1 . Therefore in the presentation we focus on this case, but we also implemented the approach for arbitrary bounded slopes. 
This paper has been organized as follows. In Section 2 we recall the earlier theory on monotone algebras and dependency pairs as we need it, and we introduce quasi-periodic functions and investigate their basic properties. In Section 3 we work out these basic ingredients towards a proof scheme for string rewriting. In Section 4 we describe how this has been implemented by transforming the corresponding search problem to a SAT problem, and investigate some experimental results. In Section 5 we describe multi-dimensional quasi-periodic functions, and investigate how to use them for extending the approach to term rewriting. In Section [6 we describe a direct approach of using quasi-periodic functions without the use of the dependency pair transformation. Finally, in Section 7 we give some conclusions.

\section{Basic Theory}

For a term rewriting system (TRS) $R$ we write $\rightarrow_{R}$ for its rewrite relation and $\stackrel{\text { top }}{\rightarrow} R$ for its top rewrite relation, i.e., $t \stackrel{\text { top }}{\rightarrow} R u$ if and only if there is a rewrite rule $\ell \rightarrow r \in R$ and a substitution $\sigma$ such that $t=\ell \sigma$ and $u=r \sigma$.

A relation $\rightarrow$ is called well-founded, terminating or strongly normalizing, notation $\mathrm{SN}(\rightarrow)$, if no infinite sequence $t_{1}, t_{2}, t_{3}, \ldots$ exists such that $t_{i} \rightarrow t_{i+1}$ for all $i=1,2,3, \ldots$ A TRS $R$ is called terminating if $\mathrm{SN}\left(\rightarrow_{R}\right)$, shortly written as $\mathrm{SN}(R)$.

A binary relation $\rightarrow_{1}$ is called terminating relative to a binary relation $\rightarrow_{2}$, written as $\mathrm{SN}\left(\rightarrow_{1} / \rightarrow_{2}\right)$, if no infinite sequence $t_{1}, t_{2}, t_{3}, \ldots$ exists such that

$-t_{i} \rightarrow_{1} t_{i+1}$ for infinitely many values of $i$, and

$-t_{i} \rightarrow_{2} t_{i+1}$ for all other values of $i$.

We use the notation $\rightarrow_{1} / \rightarrow_{2}$ to denote $\rightarrow_{2}^{*} \cdot \rightarrow_{1} \cdot \rightarrow_{2}^{*}$; it is easy to see that $\mathrm{SN}\left(\rightarrow_{1} / \rightarrow_{2}\right)$ coincides with well-foundedness of $\rightarrow_{1} / \rightarrow_{2}$. We write $\mathrm{SN}(R / S)$ as a shorthand for $\mathrm{SN}\left(\rightarrow_{R} / \rightarrow_{S}\right)$, and we write $\mathrm{SN}\left(R_{\text {top }} / S\right)$ as a shorthand for

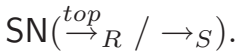

For a TRS $R$ over a signature $\Sigma$ a symbol $f \in \Sigma$ is called a defined symbol if $f$ is the root symbol of a left-hand side of a rule of $R$. For every defined symbol $f \in \Sigma$ a new marked symbol $f_{\#}$ is added having the same arity as $f$. If $f\left(s_{1}, \ldots, s_{n}\right) \rightarrow C\left[g\left(t_{1}, \ldots, t_{m}\right)\right]$ is a rule in $R$ and $g$ is a defined symbol of $R$, then the rewrite rule $f_{\#}\left(s_{1}, \ldots, s_{n}\right) \rightarrow g_{\#}\left(t_{1}, \ldots, t_{m}\right)$ is called a dependency pair of $R$. The TRS consisting of all dependency pairs of $R$ is denoted by $\operatorname{DP}(R)$.

The main theorem about dependency pairs is the following, due to Arts and Giesl, [1].

Theorem 1. Let $R$ be a TRS. Then $\operatorname{SN}(R)$ if and only if $\operatorname{SN}\left(\operatorname{DP}(R)_{\text {top }} / R\right)$.

In order to prove termination of a TRS $R$ we will prove $\operatorname{SN}\left(\operatorname{DP}(R)_{t o p} / R\right)$. In order to do so we describe a technique for proving $\operatorname{SN}\left(R_{t o p} / S\right)$ for arbitrary TRSs $R, S$, based on weakly monotone algebras. 
Definition 1. A $\Sigma$-algebra $(A,[\cdot])$ is defined to consist of a non-empty set $A$, and for every $f \in \Sigma$ a function $[f]: A^{n} \rightarrow A$, where $n$ is the arity of $f$. This function $[f]$ is called the interpretation of $f$.

$A$ operation $[f]: A^{n} \rightarrow A$ is monotone with respect to a binary relation $\rightarrow$ on $A$ if for all $a_{i}, b_{i} \in A$ for $i=1, \ldots, n$ with $a_{i} \rightarrow b_{i}$ for some $i$ and $a_{j}=b_{j}$ for all $j \neq i$ we have

$$
[f]\left(a_{1}, \ldots, a_{n}\right) \rightarrow[f]\left(b_{1}, \ldots, b_{n}\right) .
$$

$A$ weakly monotone $\Sigma$-algebra $(A,[\cdot],>, \gtrsim)$ is a $\Sigma$-algebra $(A,[\cdot])$ equipped with two relations $>, \gtrsim$ on $A$ such that

$->$ is well-founded;

$->\cdot \gtrsim \subseteq>$;

- for every $f \in \Sigma$ the operation $[f]$ is monotone with respect to $\gtrsim$.

The combination $>$, $\gtrsim$ is closely related to the notion of reduction pair as presented e.g. in [7]. A crucial difference is that the relations in a reduction pair are relations on terms that are closed under substitutions, while in our setting they are relations on the set $A$.

Writing $\mathcal{X}$ for the set of variables, for a $\Sigma$-algebra $(A,[\cdot])$ and a map $\alpha: \mathcal{X} \rightarrow A$ the term evaluation $[\cdot, \alpha]: \mathcal{T}(\Sigma, \mathcal{X}) \rightarrow A$ is defined inductively by

$$
[x, \alpha]=\alpha(x), \quad\left[f\left(t_{1}, \ldots, t_{n}\right), \alpha\right]=[f]\left(\left[t_{1}, \alpha\right], \ldots,\left[t_{n}, \alpha\right]\right)
$$

for $f \in \Sigma$ and $x \in \mathcal{X}$.

As the main property of weakly monotone algebras we recall the following theorem from [4].

Theorem 2. Let $R, S$ be TRSs over a signature $\Sigma$. Let $(A,[\cdot],>, \gtrsim)$ be a weakly monotone $\Sigma$-algebra such that $[\ell, \alpha] \gtrsim[r, \alpha]$ for every rule $\ell \rightarrow r$ in $R \cup S$ and every $\alpha: \mathcal{X} \rightarrow A$. Let $R^{\prime}$ consist of all rules $\ell \rightarrow r$ from $R$ satisfying $[\ell, \alpha]>[r, \alpha]$ for every $\alpha: \mathcal{X} \rightarrow A$.

Then $\mathrm{SN}\left(\left(R \backslash R^{\prime}\right)_{\text {top }} / S\right)$ if and only if $\mathrm{SN}\left(R_{\text {top }} / S\right)$.

The approach for proving $\mathrm{SN}(R)$ now is trying to prove $\operatorname{SN}\left(\mathrm{DP}(R)_{\text {top }} / R\right)$ by finding a suitable weakly monotone algebra such that according to Theorem 2 rules from $\mathrm{DP}(R)$ may be removed. This is repeated until all rules of $\mathrm{DP}(R)$ have been removed.

We will focus on $A=\mathbf{N}$, where $>$ is the usual ordering on $\mathbf{N}$ and $\gtrsim$ coincides with $\geq$. Now indeed $>$ is well-founded and $>\cdot \gtrsim \subseteq>$ holds, so the only requirement for being a weakly monotone algebra is that for every $f \in \Sigma$ the operation $[f]$ is monotone with respect to $\geq$. Monotonicity with respect to $\geq$ is called weak monotonicity.

Definition 2. A function $f: \mathbf{N} \rightarrow \mathbf{N}$ is called quasi-periodic with period $p \in$ $\mathbf{N}, p>0$, and slope $s \in \mathbf{N}$ if $f(x+p)=f(x)+s * p$ for all $x \in \mathbf{N}$.

For $[f]$ we will use quasi-periodic functions in case $f$ is unary; for $f$ of higher arity we will extend this notion in Section 5 . 
Theorem 3. Let $f: \mathbf{N} \rightarrow \mathbf{N}$ be a quasi-periodic function with period $p \in \mathbf{N}$, $p>0$, and slope $s \in \mathbf{N}$. Then

1. $f(x+n * p)=f(x)+n * s * p$ for all $x, n \in \mathbf{N}$.

2. $f$ is weakly monotone if and only if $f(x+1) \geq f(x)$ for $x=0, \ldots, p-1$.

3. Let $g: \mathbf{N} \rightarrow \mathbf{N}$ be a quasi-periodic function with period $p$ and slope $t$. Then $f \circ g$ is quasi-periodic with period $p$ and slope $s * t$.

4. Let $g: \mathbf{N} \rightarrow \mathbf{N}$ be a quasi-periodic function with period $p$ and slope $t$. Then $f(x) \geq g(x)$ for all $x \in \mathbf{N}$ if and only if $s \geq t$ and $f(x) \geq g(x)$ for $x=0, \ldots, p-1$.

Proof. 1. Induction on $n$.

2. By definition $f$ is weakly monotone if and only if $f(x+i) \geq f(x)$ for all $x, i \in \mathbf{N}$; by induction on $i$ this is equivalent to $f(x+1) \geq f(x)$ for all $x$. For every $x \in \mathbf{N}$ we can write $x=x^{\prime}+n * p$ for some $n \in \mathbf{N}$ with $x^{\prime}<p$. Using part (1) then yields $f(x+1)-f(x)=f\left(x^{\prime}+1\right)-f\left(x^{\prime}\right)$ from which the claim follows.

3. Using $t \in \mathbf{N}$ and part (1) we obtain

$$
\begin{aligned}
(f \circ g)(x+p) & =f(g(x+p)) \\
& =f(g(x)+t * p) \\
& =f(g(x))+s * t * p \\
& =(f \circ g)(x))+s * t * p
\end{aligned}
$$

4. For the 'if'-part we write $x=x^{\prime}+n * p$ with $x^{\prime}<p$ and use part (1):

$$
\begin{aligned}
f(x)-g(x) & =f\left(x^{\prime}\right)+n * s * p-\left(g\left(x^{\prime}\right)+n * t * p\right) \\
& =f\left(x^{\prime}\right)-g\left(x^{\prime}\right)+n *(s-t) * p \\
& \geq 0
\end{aligned}
$$

For the 'only if'-part we assume $f(x) \geq g(x)$ for all $x \in \mathbf{N}$ and need to show that $s \geq t$. Assume not, then $t \geq s+1$ and $f(n * p)-g(n * p)=$ $f(0)-g(0)+n *(s-t) * p \leq f(0)-g(0)-n * p<0$ for $n$ large enough, contradiction.

A quasi-periodic function with period $p$ is also quasi-periodic with period $n * p$ for $n \in \mathbf{N}, n>0$. Using part (3) of Theorem 3 one sees that composition of quasi-periodic functions with distinct periods is again quasi-periodic, with the period being the least common multiple of both periods. However, we will not use this: in every setting we fix all periods to be the same.

One may also allow non-integer slopes. For instance, $f$ defined by $f(x)=$ $2 *(x \operatorname{div} 3)$ can be seen as quasi-periodic with period 3 and slope $\frac{2}{3}$. However, quasi-periodic functions with a fixed period and non-integer slope are not closed under composition. For instance, for this function $f$ the function $f \circ f$ does not have period 3 any more. It has period 9 and slope $\frac{4}{9}$. Since for our purpose closedness under composition is essential we restrict to integer non-negative slopes. We allow the slope to be 0 ; this corresponds to periodic functions. A quasi-periodic function with slope 0 is weakly monotone if and only if it is constant. 


\section{$3 \quad$ String Rewriting}

String rewriting can be seen as the special case of term rewriting in which all symbols are unary. In this case we need only one variable $x$, and we identify the string $a_{1} \cdots a_{n}$ with the term $a_{1}\left(\cdots\left(a_{n}(x)\right) \cdots\right)$. We write $\alpha_{i}$ for the map mapping this variable $x$ to the number $i$, for the rest we may forget about this variable.

We propose the following approach for proving termination of an SRS $S$.

Compute $\operatorname{DP}(S)$ and try to prove $\operatorname{SN}\left(\operatorname{DP}(S)_{t o p} / S\right)$; if we succeed then indeed by Theorem 1 we may conclude $\operatorname{SN}(S)$. Trying to prove $\operatorname{SN}\left(R_{t o p} / S\right)$ for an SRS $R$ is done as follows. First try to remove rules from $R$ by Theorem 2 using simple polynomials (or any other fast technique). Next apply the following proof scheme:

\section{Proof scheme.}

- Fix a period $p \geq 1$.

- For every symbol $a$ choose a slope slope $(a)$ and $p$ natural numbers $[a](x)$ for $x=0, \ldots, p-1$, fully defining $[a]: \mathbf{N} \rightarrow \mathbf{N}$ by $[a](x+p)=[a](x)+p * \operatorname{slope}(a)$, meeting the following requirements:

- $[a](x+1) \geq[a](x)$ for every symbol $a$ and every $x=0, \ldots, p-1$ (where $[a](p)=[a](0)+p * \operatorname{slope}(a))$

- $\left[\ell, \alpha_{i}\right] \geq\left[r, \alpha_{i}\right]$ for all rules $\ell \rightarrow r$ in $R \cup S$ and all $i=0, \ldots, p-1$,

- for at least one rule $\ell \rightarrow r$ in $R$ it holds that $\left[\ell, \alpha_{i}\right]>\left[r, \alpha_{i}\right]$ for all $i=0, \ldots, p-1$; write $R^{\prime}$ for the rules from $R$ for which this holds.

- If $R^{\prime}=R$ we are done, otherwise $\mathrm{SN}\left(\left(R \backslash R^{\prime}\right)_{\text {top }} / S\right)$ has to be proved, either by repeating this proof scheme or by any other technique.

Correctness of this approach follows by combining Theorem 2 and Theorem 3 . For $p=1$ the scheme coincides with linear polynomials.

Example 1. We consider the example mentioned in the introduction: the two rules $a a a \rightarrow b a b, \quad b b b \rightarrow a a a$. Applying Theorem 1 we will prove termination of this SRS $S$ by proving $\operatorname{SN}\left(\operatorname{DP}(S)_{t o p} / S\right)$. By considering lengths of strings, more precisely, by applying Theorem 2 where the algebra consists of the natural numbers and every symbol is interpreted by the successor function, it remains to prove $\mathrm{SN}\left(R_{t o p} / S\right)$ for $R$ consisting of the two rules $A a a \rightarrow B a b, B b b \rightarrow A a a$, where we simply write $A, B$ instead of the marked symbols $a_{\#}$ and $b_{\#}$. For this we apply the above approach: we fix $p=3$, all slopes are one, and choose

$$
\begin{array}{llll}
{[a](0)=2,} & {[b](0)=3,} & {[A](0)=2,} & {[B](0)=3,} \\
{[a](1)=3,} & {[b](1)=3,} & {[A](1)=4,} & {[B](1)=4,} \\
{[a](2)=4,} & {[b](2)=3,} & {[A](2)=5,} & {[B](2)=4 .}
\end{array}
$$

All properties are checked for $R^{\prime}$ consisting of the rule $B b b \rightarrow A a a$. So this rule may be removed from $R$. The remaining property $\operatorname{SN}\left(\{A a a \rightarrow B a b\}_{t o p} / S\right)$ is easily proved by counting the number of $A$ symbols. 
We conclude this section by some basic transformations on SRSs preserving termination.

For a string $s$ write $s^{\text {rev }}$ for its reverse. For an SRS $R$ write

$$
R^{\mathrm{rev}}=\left\{\ell^{\mathrm{rev}} \rightarrow r^{\mathrm{rev}} \mid \ell \rightarrow r \in R\right\}
$$

and

$$
R^{-1}=\{r \rightarrow \ell \mid \ell \rightarrow r \in R\} .
$$

From [13] we recall the following simple lemma.

Lemma 1. Let $R$ be an $S R S$.

1. $\mathrm{SN}(R)$ if and only if $\mathrm{SN}\left(R^{\mathrm{rev}}\right)$.

2. Assume that $R$ is finite and that for every rule $\ell \rightarrow r$ of $R$ the lengths of $\ell$ and $r$ are equal. Then $\mathrm{SN}(R)$ if and only if $\mathrm{SN}\left(R^{-1}\right)$.

Slightly more involved, and increasing the SRS size, is the following transformation. For a set $\Sigma$ of symbols we define lab : $\Sigma^{*} \times \Sigma \rightarrow(\Sigma \times \Sigma)^{*}$ as follows:

$$
\operatorname{lab}(\epsilon, a)=\epsilon, \quad \operatorname{lab}(s a, b)=\operatorname{lab}(s, a)(a, b),
$$

for all $a, b \in \Sigma, s \in \Sigma^{*}$. Here $\epsilon$ denotes the empty string. For an SRS $R$ over $\Sigma$ we define

$$
\operatorname{lab}(R)=\{\operatorname{lab}(\ell, a) \rightarrow \operatorname{lab}(r, a) \mid \ell \rightarrow r \in R \wedge a \in \Sigma\}
$$

For a non-empty string $s$ write $s_{1}$ for its first element. In order to force that for every rule $\ell \rightarrow r$ we have $\ell_{1}=r_{1}$, for arbitrary SRS $R$ over $\Sigma$ we define the SRS $F(R)$ to be

$\left\{\ell \rightarrow r \in R \mid r \neq \epsilon \wedge \ell_{1}=r_{1}\right\} \cup\left\{a \ell \rightarrow a r \mid a \in \Sigma \wedge \ell \rightarrow r \in R \wedge\left(r=\epsilon \vee \ell_{1} \neq r_{1}\right)\right\}$

Lemma 2. Let $R$ be an $S R S$. Then $\operatorname{SN}(R)$ if and only if $\operatorname{SN}(\operatorname{lab}(F(R)))$.

Proof. Equivalence of $\mathrm{SN}(R)$ and $\mathrm{SN}(F(R))$ is straightforward. Equivalence of $\mathrm{SN}(F(R))$ and $\mathrm{SN}(\operatorname{lab}(F(R)))$ is a direct application of semantic labelling [1] in which the model is $\Sigma$ and every symbol is interpreted by its own value. For the model requirement it is essential that for every rule $\ell \rightarrow r$ in $F(R)$ we have $\ell_{1}=r_{1}$.

The idea of these transformations is that if proving termination of the original system fails, then proving termination of the transformed system is tried. This idea is not new. For rev it is extensively used in several tools; for lab $(F(\cdot))$ it was extensively and very successfully used in the 2006 competition by Jambox, see 9. In Example 3 we will see how Lemma 2 can be applied fruitfully. 


\section{Implementation and Results}

We experimented with various ways for automatically finding termination proofs based on the above given proof scheme. The first one simply chose several times randomly among a class of quasi-periodic functions until all requirements were fulfilled. The class of quasi-periodic functions consisted of functions of the shape $\lambda x \cdot x+n$ and $\lambda x \cdot p *(x \operatorname{div} p)+n$ for constants $n$. In this way the first automatically found termination proof for the example in the introduction was given, only a few days after Nogin and Witty found their manual proof.

However, this random search has a few drawbacks:

- It depends on the special choice of the shape of the quasi-periodic functions chosen, being much more restricted than arbitrary quasi-periodic functions with fixed period and slope.

- If no proof is found you do not know whether no proof of the desired shape exists or you did not yet try long enough.

A remedy against both these drawbacks is the following. For all numbers chosen in the proof scheme choose numbers in binary notation, and introduce boolean variables for each of the bits. Express all requirements in the proof scheme as propositional requirements on these boolean variables. Then a choice of the numbers satisfying all requirements is possible if and only if the formula is satisfiable. So the approach is to apply a state-of-the-art SAT solver to the resulting formula (just like for several other methods for proving termination), and in case the formula is satisfiable transform the bits of the numbers in the corresponding satisfying assignment back to the numbers they represent.

Still the encoding of the requirements can be done in several ways. One fruitful way is the following. It easily extends to arbitrary slopes; for keeping the presentation simple we restrict here to the case where all slopes are one. Assume we want to prove $\mathrm{SN}\left(R_{t o p} / S\right)$.

We fix three numbers: $p$ is the period, $n$ is the number of bits per number, by which all numbers are non-negative and $<2^{n}$, and $m$ is the maximal number allowed as an intermediate result, satisfying $p<m<2^{n}-p$. For all symbols $a$ we choose $m * n$ boolean variables for the $m n$-bit numbers $[a](0), \ldots,[a](m-1)$. For these we generate the requirements $[a](i) \leq[a](i+1)$ for $i=0, \ldots, p-1$ and $[a](i+p)=[a](i)+p$ for $i=0, \ldots, m-p-1$. Having all these numbers $[a](0), \ldots,[a](m-1)$ available in separate $n$-bit notation makes it straightforward to express the boolean formula $[a](i)=j$ for given numbers $i, j, i<m$.

In order to check $\left[\ell, \alpha_{i}\right] \geq\left[r, \alpha_{i}\right]$, for every rule $\ell \rightarrow r$ in $R \cup S$ and every $i=0, \ldots, p-1$ the following is done. Write $\ell=a_{1} a_{2} \cdots a_{k}$, then $k$ fresh binary numbers $\ell_{i, 1}, \ldots, \ell_{i, k}$ are introduced, for which the following requirements are created:

$$
\begin{gathered}
\qquad \ell_{i, k}=\left[a_{k}\right](i), \\
\left(j=\ell_{i, q}\right) \rightarrow\left(\ell_{i, q-1}=\left[a_{q}\right](j)\right) \text {, for all } q=1, \ldots, k, j=0, \ldots, m-1, \\
m>\ell_{i, q}, \text { for all } q=2, \ldots, k .
\end{gathered}
$$


This is done similarly for $r$. Next the requirement $\ell_{i, 1} \geq r_{i, 1}$ is generated. Finally the requirement is added that for at least one rule in $R$ we have $\ell_{i, 1}>r_{i, 1}$.

A remarkable property of this approach is that the only arithmetic that occurs in these formulas is the unary function $\lambda x \cdot x+p$ and checking for $>$ and $\geq$. As a consequence, the resulting formulas are relatively small. For instance, our solution given in Example 1 was found by applying SAT on a formula consisting of 6065 clauses on 872 variables, in a fraction of a second, after choosing the parameters $p=3, n=4, m=7$.

In order to find termination proofs fully automatically, the approach should not depend on parameters that have to be set manually. Therefore we need heuristics for setting these parameters and for how to combine this with other techniques.

We chose always to combine this with basic polynomials, more precisely, apply Theorem 2 with natural numbers and linear polynomials with coefficients in $n$ bits, and try both these basic polynomials and the quasi-periodic interpretations with the fixed parameters as long as possible. Moreover, we apply Lemma 1; after an attempt fails for an SRS $S$, then a next attempt is done for $S^{\text {rev }}$. If even that fails, then for length-preserving systems the same is done for $S^{-1}$.

Of course our approach is easily combined with other termination techniques, but in order to investigate the merits of the technique itself we concentrated on running the experiments in this most basic setting.

After fixing this basic setting, a choice should be made for the parameters $p, n, m$. We experimented with these parameters on several small SRSs for which termination was unknown until now.

It appears that increasing these parameters never decreases the power. For the parameters $n, m$ this is obvious; for the period $p$ this is only indicated by experiments: we failed to prove this. For one system, Gebhardt18 in TPDB, consisting of the two rules $0000 \rightarrow 1011,1001 \rightarrow 0000$, it turned out that no proof was found with period less than 5 , but a proof was found for period 5 . We did not find examples that could be solved with period higher than 5 but not by period 5. So a suitable choice for the period is 5 .

It turned out that all proofs we found could be found using numbers of four bits. A corresponding choice of the parameters is $n=4$ and $m=10$. Fixing these parameters $p=5, n=4$ and $m=10$ we found termination proofs of the following 11 SRSs in TPDB 2006, of which in the 2006 competition none of the participating tools found a termination proof:

in the directory Endrullis: systems 01, 02, 05 and 06, in the directory Gebhardt: systems 01, 04, 07, 11, 17 and 18, in the directory Waldmann: system jw1 (this is the system from Example 1).

All of these systems are very small: each one consists of only two rules over two symbols. In all of these cases finding the satisfying assignment of the SAT formula representing the quasi-periodic interpretation was done by minisat within a fraction of a second.

Example 2. As an example we give the proof of the system Gebhardt18 as shown above, found in this way by our implementation with the parameters $p=5, n=4$ 
and $m=10$. After removing the length-decreasing dependency pairs it remains to prove $\operatorname{SN}\left(R_{\text {top }} / S\right)$ for $R$ consisting of the rules $0_{\#} 000 \rightarrow 1_{\#} 011,1_{\#} 001 \rightarrow$ $0_{\#} 000$ and $S$ consisting of the original rules $0000 \rightarrow 1011,1001 \rightarrow 0000$. For that the following quasi-periodic interpretation with period 5 is found:

$$
\begin{aligned}
{[0](0) } & =1, \quad[0](1)=2, \quad[0](2)=3, \quad[0](3)=4, \quad[0](4)=5, \\
{[1](0) } & =1,[1](1)=1, \quad[1](2)=1, \quad[1](3)=6, \quad[1](4)=6, \\
{\left[0_{\#}\right](0) } & =2,\left[0_{\#}\right](1)=7,\left[0_{\#}\right](2)=7,\left[0_{\#}\right](3)=7,\left[0_{\#}\right](4)=7, \\
{\left[1_{\#}\right](0) } & =4,\left[1_{\#}\right](1)=4,\left[1_{\#}\right](2)=4,\left[1_{\#}\right](3)=8,\left[1_{\#}\right](4)=8 .
\end{aligned}
$$

As a consequence the rule $1_{\#} 001 \rightarrow 0_{\#} 000$ may be removed from $R$, after which the rest is trivial by counting the number of $0_{\#}$ symbols.

This implementation restricts to the case where all slopes are equal to one. The implementation easily extends to setting with arbitrary slopes. We also experimented with other encodings of the problem, including general slopes. Surprisingly, using general slopes we only found termination proofs for systems for which also proofs with slope one were found. Hence generalizing the slope does not seem to increase the power of the method, and therefore we do not describe the implementation for general slopes in more detail.

Example 3. As an example of a combination with other techniques we consider the special case of semantic labelling (11]) as described in Lemma 2. Applying $\operatorname{lab}(F(\cdot))$ to the SRS Gebhardt09, consisting of the two rules $0000 \rightarrow 0111$, $1001 \rightarrow 0000$, yields the transformed system of six rules over the four symbols 00, 01, 10 and 11:

$$
\begin{array}{rlrl}
00000000 & \rightarrow 01111110, & 00000001 & \rightarrow 01111111, \\
0110000110 & \rightarrow 0000000000, & 0110000111 \rightarrow 0000000001, \\
1110000110 & \rightarrow 1000000000, & 1110000111 \rightarrow 1000000001 .
\end{array}
$$

It turns out that by the technique described in this paper termination of this transformed SRS (and hence of the original SRS by Lemma 2) is easily proved, again automatically generating a termination proof of an SRS for which termination was open until now.

A similar termination proof was found for the SRS Waldmann-sym-5, consisting of the two rules $a a a \rightarrow b b b, \quad b b b b \rightarrow a b b a$. For this SRS termination was open until now too, by which the total score of new automatic proofs for unsolved SRSs from TPDB becomes 13 .

\section{Term Rewriting}

In order to apply our method to term rewriting, we call a function $f: \mathbf{N}^{k} \rightarrow \mathbf{N}$ quasi-periodic with period $p \in \mathbf{N}, p>0$ and slope $s=\left(s_{1}, \ldots, s_{k}\right) \in \mathbf{N}^{k}$ if for each $x \in \mathbf{N}^{k}$ and each $1 \leq i \leq k$

$$
f\left(x_{1}, \ldots, x_{i}+p, \ldots, x_{k}\right)=f\left(x_{1}, \ldots, x_{i}, \ldots, x_{k}\right)+s_{i} * p .
$$

That is, $f$ is quasi-periodic with slope $s_{i}$ in its $i$-th argument. 
The analogue of Theorem 3 still holds, with similar proof:

Theorem 4. Let $f: \mathbf{N}^{k} \rightarrow \mathbf{N}$ be a quasi-periodic function with period $p \in$ $\mathbf{N}, p>0$ and slope $s=\left(s_{1}, \ldots, s_{k}\right) \in \mathbf{N}^{k}$. Then

1. $f$ is weakly monotonic if for all $\left(x_{1}, \ldots, x_{k}\right) \in\{0,1, \ldots, p-1\}^{k}$ and for all $1 \leq i \leq k$ we have $f\left(x_{1}, \ldots, x_{i}+1, \ldots, x_{k}\right) \geq f\left(x_{1}, \ldots, x_{i}, \ldots, x_{k}\right)$.

2. Let $g_{1}, \ldots, g_{k}: \mathbf{N}^{l} \rightarrow \mathbf{N}$ be quasi-periodic functions with period $p$ and slopes $t_{1}, \ldots, t_{k}$, respectively. Then the combined function $h: \mathbf{N}^{l} \rightarrow \mathbf{N}$ defined by $h(x)=f\left(g_{1}(x), \ldots, g_{k}(x)\right)$ is quasi-periodic with period $p$ and its slope in the $i$-th position is $s_{1} t_{1, i}+\ldots+s_{k} t_{k, i}$.

3. Let $g: \mathbf{N}^{k} \rightarrow \mathbf{N}$ be a quasi-periodic function with period $p$ and slope $t=$ $\left(t_{1}, \ldots, t_{k}\right)$. Then $f(x) \geq g(x)$ for all $x \in \mathbf{N}^{k}$ if and only if $s_{1} \geq t_{1}, \ldots, s_{k} \geq$ $t_{k}$ and $f(x) \geq g(x)$ for all $x \in\{0,1, \ldots, p-1\}^{k}$.

Corollary 1. If an interpretation [.] is given that assigns to each $k$-ary function symbol $f$ from the signature $\Sigma$ a $k$-ary quasi-periodic function $[f]$ with period $p$, then the interpretation $[t]$ of any term $t$ containing $n$ variables is an $n$-ary quasi-periodic function with period $p$.

Proof. If $t$ is a variable $v_{i}$, then $[t]$ is the projection $\left(v_{1}, \ldots, v_{n}\right) \mapsto v_{i}$ which is quasi-periodic with slope $(0, \ldots, 0,1,0 \ldots 0)$ and any period $p>0$. If $t=$ $f\left(t_{1}, \ldots, t_{k}\right)$, then the interpretation can be composed (by Theorem 4) from the interpretations $\left[t_{1}\right], \ldots,\left[t_{k}\right]$. This includes the case $k=0$.

To find a quasi-periodic interpretation that proves (relative) termination of a given term rewriting system, we extend the SAT solver approach mentioned in Section 4. In the following, the word variable means "a sequence of Boolean variables that represents a natural number".

We represent a $k$-ary quasi-periodic function $f$ of period $p$ by $k$ variables that represent the slopes $\left(s_{1}, \ldots, s_{k}\right)$ and $p^{k}$ variables that represent $f(x)$ for $x \in\{0,1, \ldots, p-1\}^{k}$. We ensure that $f$ is weakly monotonic by $p^{k} \times k$ constraints according to Theorem 4 , part 1 .

With the notation of Part 2 of that theorem, the combined function $h$ has each component of its slope vector constrained by one equation. To constrain the (initial) values of $h$, for each argument tuple $x \in\{0, \ldots, p-1\}^{l}$, and each $i \in\{1, \ldots, k\}$, we use a pair of variables $\left(q_{i}, r_{i}\right)$ (quotient and remainder) that fulfill $g_{i}(x)=p * q_{i}+r_{i} \wedge r_{i}<p$. Then the values of $h$ must obey the constraint $h(x)=s_{1} q_{1}+\ldots+s_{k} q_{k}+f\left(r_{1}, \ldots, r_{k}\right)$.

Our implementation is restricted to the case where the period $p$ is a power of two because then the construction of $q_{i}, r_{i}$ is much simpler, since all variables use a base two representation.

We illustrate the method by the following examples.

Example 4. From the rewriting system

$$
\begin{aligned}
R=\{ & c(f(y, b(f(0, x)))) \rightarrow f(c(c(a(f(y, x)))), y), \\
& \quad f(0, b(f(y, x))) \rightarrow b(b(y)), f(y, a(f(0, x))) \rightarrow y), a(c(y)) \rightarrow y\},
\end{aligned}
$$


the essential part of the DP transformed problem is $\mathrm{SN}\left(S_{\text {top }} / R\right)$ with $S=\{C(f(y, b(f(0, x)))) \rightarrow C(c(a(f(y, x)))), C(f(y, b(f(0, x)))) \rightarrow C(a(f(y, x)))\}$.

Now we use a quasi-periodic interpretation of period 2.

$$
\begin{aligned}
& {[0]=0, \quad[a](x)=\lfloor x\rfloor_{2}, \quad[b](x)=0} \\
& {[c](x)=\lfloor x+1\rfloor_{2}, \quad[f](x, y)=\lfloor x\rfloor_{2}+1, \quad[C](x)=x .}
\end{aligned}
$$

Here, $\lfloor x\rfloor_{m}$ denotes $m *(x \operatorname{div} m)=x-(x \bmod m)$ (round down to the next multiple of $m$ ). This interpretation is weakly compatible with $R$ (meaning that $[\ell, \alpha] \geq[r, \alpha]$ for all $\alpha$ and all rules $\ell \rightarrow r)$, for instance, the interpretation of $a(c(y))$ is $\lfloor y+1\rfloor_{2}$, which is equal to $y$ for even $y$ and greater than $y$ for odd $y$; and strictly compatible with $S$ (meaning that $[\ell, \alpha]>[r, \alpha]$ for all $\alpha$ and all rules $\ell \rightarrow r)$, for instance, the interpretation of $C(f(y, b(f(0, x))))$ is $\lfloor y\rfloor_{2}+1$ and the interpretation of $C(a(f(y, x)))$ is $\lfloor y\rfloor_{2}$.

Example 5. For the rewriting system

$$
\begin{aligned}
R=\{a(c(a(c(c(f(y, 0)))))) \rightarrow y, \quad f(y, f(0, c(x))) \rightarrow a(c(y)), & \rightarrow(f(f(f(y, y), x), 0))\}
\end{aligned}
$$

the essential part of $\operatorname{DP}(R)$ is

$$
\begin{aligned}
S=\{ & F(y, f(0, c(x))) \rightarrow C(y), \quad F(y, x) \rightarrow\{C(c(y)), C(y)\}, \\
& C(a(f(y, f(0, x)))) \rightarrow\{F(f(f(y, y), x), 0), F(f(y, y), x), F(y, y)\}\},
\end{aligned}
$$

where we have grouped together rules with identical left-hand sides. Then the following quasi-periodic interpretation

$$
\begin{aligned}
& {[0]=0,[a](x)=x+1,[c](x)=\lfloor x\rfloor_{2},[C](x)=\lfloor x\rfloor_{2}+2,} \\
& {[f](x, y)=\lfloor x\rfloor_{2}+1,[F](x, y)=\lfloor x\rfloor_{2}+2}
\end{aligned}
$$

is weakly compatible with $R \cup S$ and strictly compatible with those three rules from $S$ that have $C$ as top symbol. So they can be removed, and the remaining problem is easily solved.

None of the above examples can be solved by Jambox or Aprove (2006 version). In our implementation the actual construction and solution (by minisat) of the constraint system only takes a few seconds.

Both systems in the above examples are non-linear. A quasi-periodic interpretation that is compatible with a non-linear system cannot have all slopes equal to one. The examples also indicate that it is quite powerful to extend the range of slopes to just $\{0,1\}$.

The dependency pairs transformation creates rewriting systems that consist of groups of rules with identical left-hand sides. So the computation of the interpretation of left-hand sides can be shared. In fact, our implementation also shares interpretations of identical subterms (in all rules). This leads to a substantial reduction of the size of the constraint system and the run time of the solver. 


\section{Direct Interpretations}

Until now the dependency pairs transformation is an essential ingredient of our approach. This has a few drawbacks: in this way the approach does not apply for relative termination, and neither serves for investigation of derivational complexity or more restricted variants of termination like total termination. Therefore in this section we investigate how quasi-periodic interpretations can be used directly without dependency pairs transformation. For that we need strict monotonicity rather than weak monotonicity, and the classical monotone algebra approach [12. A quasi-periodic function $f: \mathbf{N} \rightarrow \mathbf{N}$ is called strictly monotonic if $x<y$ implies $f(x)<f(y)$.

Note that a strictly monotonic quasi-periodic function has slope $\geq 1$, and the only such functions of slope $=1$ are the functions $x \mapsto x+c$. The function $x \mapsto 1+x+\lfloor x\rfloor_{2}$ is strictly monotonic and has slope 2 and period 2. Here again $\lfloor x\rfloor_{m}$ denotes $m *(x \operatorname{div} m)$. We have the following properties:

Proposition 1. - A quasi-periodic function $f$ of period $p$ is strictly monotonic if and only if we have $f(x)<f(x+1)$ for every $x \in\{0,1, \ldots, p-1\}$.

- If both $f$ and $q$ are strictly monotonic and quasi-periodic of period $p$, then the composition $x \mapsto f(g(x))$ is strictly monotonic.

This means that we can handle strictly monotonic quasi-periodic functions effectively.

The classical monotone algebra approach restricting to natural numbers states that for proving $\mathrm{SN}(R / S)$ it suffices to give an interpretation [.] that assigns to each letter of the signature a strictly monotonic function, such that $[\ell](x)>[r](x)$ for each rule $\ell \rightarrow r \in R$ and $[\ell](x) \geq[r](x)$ for each rule $\ell \rightarrow r \in S$, for all $x \in \mathbf{N}$.

This scheme can be implemented in the same way as for weakly monotonic functions. This approach may give very simple termination proofs.

Example 6. Consider the strictly monotonic interpretation of period 2

$$
[a](x)=1+x+\lfloor x\rfloor_{2}, \quad[b](x)=2 x .
$$

It is strictly compatible with $R=\left\{a^{3} \rightarrow b b a, a b a \rightarrow b b a\right\}$ and weakly compatible with $S=\{b a b \rightarrow a a b\}$. This can be checked by a finite case analysis:

\begin{tabular}{c|c|c|c|c|c}
$x$ & {$\left[a^{3}\right](x)$} & {$[a b a](x)$} & {$[b b a](x)$} & {$[b a b](x)$} & {$[a a b](x)$} \\
\hline 0 & 5 & 5 & 4 & 2 & 2 \\
1 & 10 & 9 & 8 & 10 & 10
\end{tabular}

This proves $\mathrm{SN}(R / S)$. We also have $\mathrm{SN}(S)$, by counting letters $a$, therefore $\mathrm{SN}(R \cup S)$, which is the SRS Bouchare-06 in TPDB.

This problem has been solved in the 2006 competition by some of the tools, but all generated proofs are much more complicated than this simple proof.

Since any quasi-periodic function is bounded from above by a linear function, the interpretation of a word then is bounded by an iterated linear function, 
giving an exponential function. Thus if a strictly monotonic quasi-periodic interpretation is compatible with a rewriting system (removes all rules at the same time), then the derivational complexity of that system is at most exponential. We further note that since our interpretation domain is $(\mathbf{N},>)$, which is totally ordered, we may conclude total termination. This remains true if a proof is given by repeated application of this direct method, since the lexicographic product of a number of copies of $\mathbf{N}$ is still totally ordered. Hence Example 6 shows total termination of the SRS Bouchare-06 in TPDB.

\section{Conclusions}

We introduced a new technique for proving termination of both term rewriting and string rewriting. For both categories we succeeded in giving examples where our technique applies and earlier techniques fail.

In particular, by our technique we proved termination of several SRSs in TPDB for which termination was open until now. All of them consist of only two rules over two symbols. The reason for this is simple: most of the SRSs in TPDB for which termination is open came out of extensive search among randomly generated SRSs of this shape, filtered by failure of earlier tools.

This does not mean that restricted to string rewriting our approach is only successful for SRSs of this very special shape. For instance, our approach easily finds a termination proof for the SRS consisting of the following ten rules

$$
\begin{gathered}
a b \rightarrow c d, \quad c c \rightarrow b d, \quad b \rightarrow c f, \quad d d \rightarrow g, \quad c d \rightarrow h, \\
f \rightarrow g, \quad f \rightarrow d d, \quad g h \rightarrow a c, \quad h g \rightarrow f, \quad a \rightarrow d c,
\end{gathered}
$$

where all other approaches until now fail.

In order to investigate the primary merits of our approach we focused our experiments on the most basic setting: only combine it with the most basic kind of polynomials and, for string rewriting, reversal of rules. For this basic setting we showed that our approach is successful: we found termination proofs of 11 systems in TPDB for which termination was open until now. This is definitely not the end point: this shows that it makes sense to plug in our technique in other tools for proving termination, in order to combine it with the wide range of termination techniques that have been implemented until now. Of course we appreciate that this will not be done before publication of this paper. As a first step in this direction we showed that by combining our approach with a very basic instance of semantic labelling for string rewriting, termination could be proved of two more SRSs in TPDB for which termination was open until now.

\section{References}

1. Arts, T., Giesl, J.: Termination of term rewriting using dependency pairs. Theoretical Computer Science 236, 133-178 (2000)

2. Termination Problems Data Base http://www.lri.fr/ marche/tpdb/ 
3. Eén, N., Biere, A.: Effective preprocessing in SAT through variable and clause elimination. In: Bacchus, F., Walsh, T. (eds.) SAT 2005. LNCS, vol. 3569, pp. 6175. Springer, Heidelberg (2005) Tool/http://www.cs.chalmers.se/Cs/Research/FormalMethods/MiniSat/

4. Endrullis, J., Waldmann, J., Zantema, H.: Matrix interpretations for proving termination of term rewriting. In: Furbach, U., Shankar, N. (eds.) IJCAR 2006. LNCS (LNAI), vol. 4130, pp. 574-588. Springer, Heidelberg (2006)

5. Geser, A.: Termination of string rewriting rules that have one pair of overlaps. In: Nieuwenhuis, R. (ed.) RTA 2003. LNCS, vol. 2706, pp. 410-423. Springer, Heidelberg (2003)

6. Geser, A., Hofbauer, D., Waldmann, J., Zantema, H.: Finding finite automata that certify termination of string rewriting. International Journal of Foundations of Computer Science 16(3), 471-486 (2005)

7. Giesl, J., Arts, T., Ohlebusch, E.: Modular termination proofs for rewriting using dependency pairs. Journal of Symbolic Computation 34(1), 21-58 (2002)

8. Hofbauer, D., Waldmann, J.: Proving termination with matrix interpretations. In: Pfenning, F. (ed.) RTA 2006. LNCS, vol. 4098, pp. 328-342. Springer, Heidelberg (2006)

9. Marché, C., Zantema, H.: The termination competition. In: Baader, F. (ed.) Proceedings of the 18th Conference on Rewriting Techniques and Applications (RTA), Springer, Heidelberg (2007) http://www.lri.fr/ marche/termination-competition/

10. Moczydłowski, W., Geser, A.: Termination of single-threaded one-rule Semi-Thue systems. In: Giesl, J. (ed.) RTA 2005. LNCS, vol. 3467, pp. 338-352. Springer, Heidelberg (2005)

11. Zantema, H.: Termination of term rewriting by semantic labelling. Fundamenta Informaticae 24, 89-105 (1995)

12. Zantema, H.: Termination. In: Term Rewriting Systems, by Terese, pp. 181-259. Cambridge University Press, Cambridge (2003)

13. Zantema, H.: Termination of string rewriting proved automatically. Journal of Automated Reasoning 34, 105-139 (2004)

14. Zantema, H., Geser, A.: A complete characterization of termination of $0^{p} 1^{q} \rightarrow 1^{r} 0^{s}$. In: Hsiang, J. (ed.) Rewriting Techniques and Applications. LNCS, vol. 914, pp. 41-55. Springer, Heidelberg (1995) 